\title{
Improving the magnetodynamical properties of NiFe/Pt bilayers through $\mathrm{Hf}$ dusting
}

Hamid Mazraati, Mohammad Zahedinejad, and Johan Åkerman

Citation: Appl. Phys. Lett. 113, 092401 (2018); doi: 10.1063/1.5026232

View online: https://doi.org/10.1063/1.5026232

View Table of Contents: http://aip.scitation.org/toc/apl/113/9

Published by the American Institute of Physics

\section{Articles you may be interested in}

Temperature dependence of the Dzyaloshinskii-Moriya interaction in $\mathrm{Pt} / \mathrm{Co} / \mathrm{Cu}$ thin film heterostructures Applied Physics Letters 113, 092402 (2018); 10.1063/1.5038353

Dual-mode ferromagnetic resonance in an $\mathrm{FeCoB} / \mathrm{Ru} / \mathrm{FeCoB}$ synthetic antiferromagnet with uniaxial anisotropy Applied Physics Letters 112, 192401 (2018); 10.1063/1.5018809

Structural, magnetic, and transport properties of $\mathrm{Fe}_{1-x} \mathrm{Rh}_{\mathrm{X}} / \mathrm{MgO}(001)$ films grown by molecular-beam epitaxy Applied Physics Letters 113, 082403 (2018); 10.1063/1.5048303

Direct detection of spin Nernst effect in platinum

Applied Physics Letters 112, 162401 (2018); 10.1063/1.5021731

Spin-orbit torques in high-resistivity-W/CoFeB/MgO

Applied Physics Letters 112, 192408 (2018); 10.1063/1.5027855

Temperature dependence of interlayer exchange coupling and Gilbert damping in synthetic antiferromagnetic trilayers investigated using broadband ferromagnetic resonance

Applied Physics Letters 113, 042401 (2018); 10.1063/1.5040666

\section{Conference Proceedings}




\title{
Improving the magnetodynamical properties of NiFe/Pt bilayers through Hf dusting
}

\author{
Hamid Mazraati, ${ }^{1,2}$ Mohammad Zahedinejad, ${ }^{3}$ and Johan Åkerman ${ }^{1,2,3, a)}$ \\ ${ }^{1}$ NanOsc AB, Kista 164 40, Sweden \\ ${ }^{2}$ Department of Applied Physics, School of Engineering Sciences, KTH Royal Institute of Technology, \\ 16400 Kista, Sweden \\ ${ }^{3}$ Department of Physics, University of Gothenburg, 41296 Gothenburg, Sweden
}

(Received 16 February 2018; accepted 15 August 2018; published online 29 August 2018)

\begin{abstract}
We investigate the effect of hafnium (Hf) dusting on the magnetodynamical properties of $\mathrm{NiFe} / \mathrm{Pt}$ bilayers using spin-torque-induced ferromagnetic resonance measurements on $6 \mu \mathrm{m}$ wide microstrips on high-resistive Si substrates. Based on two series of $\mathrm{NiFe}\left(t_{\mathrm{NiFe}}\right) / \mathrm{Hf}\left(t_{\mathrm{Hf}}\right) / \mathrm{Pt}(5)$ stacks, we first demonstrate that the zero-current magnetodynamic properties of the devices benefit from Hf dusting: (i) the effective magnetization of the NiFe layer increases by $4 \%-8 \%$ with Hf present and (ii) the damping $\alpha$ decreases linearly with $t_{\mathrm{Hf}}$ by up to $40 \%$. The weaker anisotropic magnetoresistance $(A M R \simeq 0.3 \%-0.4 \%)$ of the $3 \mathrm{~nm} \mathrm{NiFe}$ series is largely unaffected by the Hf, while the stronger AMR of the $5 \mathrm{~nm} \mathrm{NiFe}$ series drops from $0.7 \%$ to $0.43 \%$ with increasing $t_{\mathrm{Hf}}$. We find that the spin Hall efficiency $\xi_{\mathrm{SH}}$ is independent of the NiFe thickness, remaining unaffected $\left(\xi_{\mathrm{SH}}=0.115\right)$ up to $t_{\mathrm{Hf}}=0.4 \mathrm{~nm}$ and then decreasing linearly for higher $t_{\mathrm{Hf}}$. The different trends of $\alpha$ and $\xi_{\mathrm{SH}}$ suggest that there is an optimum Hf thickness $(\simeq 0.4 \mathrm{~nm})$ for which the threshold current for auto-oscillation should have a minimum, while the much lower damping should improve mutual synchronization. Our results also indicate that the spin-orbit torque is entirely damping-like with no field-like torque component. Finally, the internal spin Hall angle of Pt is estimated to be $\theta_{\mathrm{SH}}=0.22$ by calculating the transparency of the interface. Published by AIP Publishing.

https://doi.org/10.1063/1.5026232
\end{abstract}

The spin Hall effect (SHE) is the generation of a pure transverse spin current $J_{\mathrm{s}}$ proportional to a lateral charge current $J_{\mathrm{e}}$ flowing through certain heavy metals (HMs), with a proportionality constant, $\theta_{\mathrm{SH}}$, referred to as the spin Hall angle. ${ }^{1-5}$ The SHE represents a promising route towards spin-current-based microwave signal generators-so-called spin Hall nano-oscillators (SHNOs). ${ }^{6-10}$ These SHNOs generally consist of a heavy metal (HM)/ferromagnetic (FM) bilayer where the spin current from SHE can be absorbed by the FM layer, generating a spin-orbit torque on its magnetization. The efficiency of this torque, $\xi_{\mathrm{SH}}$, is defined as the ratio of the absorbed current to the charge current and is always smaller than $\theta_{\mathrm{SH}} \cdot{ }^{11}$ Besides the generation of microwave signals, SHNOs have also been considered for use as microwave detectors ${ }^{12}$ and modulators ${ }^{13}$ and even in neuromorphic computing applications. ${ }^{14}$

For most of these applications, the SHNO performance needs to be enhanced to offer low threshold currents (low power consumption), high microwave output power, and low linewidth. While it has been shown that the synchronization of two or more SHNOs can be used to improve the power and coherency of the generated microwave signal, ${ }^{15-17} \mathrm{sev}-$ eral studies have also focused on enhancing the efficiency of spin current generation by using other heavy metals with higher $\xi_{\mathrm{SH}}$, such as $\beta-\mathrm{W},{ }^{18-20} \beta-\mathrm{Ta},{ }^{21}$ and $\mathrm{V} .{ }^{22}$ Compared to $\mathrm{Pt}$, these materials typically suffer from excessive heating due to their higher resistivity, which can be problematic when a large number of them are placed together in a small

\footnotetext{
${ }^{\text {a) }}$ Author to whom correspondence should be addressed: johan.akerman@ physics.gu.se
}

area to operate collectively, increasing the proportion of the current going through the ferromagnetic layer.

On the other hand, $\xi_{\mathrm{SH}}$ for Pt is not as high as for other candidates, and $\mathrm{Pt}$ also appears to have a more detrimental effect on the zero-current damping of the Pt/FM bilayer. Recent work $^{23-25}$ has however shown both a reduction in this damping and an enhancement in the efficiency of SHE in Pt through careful engineering of the Pt/FM interface: In one work, ${ }^{25}$ a very thin hafnium (Hf) layer was inserted between the $\mathrm{Pt}$ and $\mathrm{CoFeB}$ in order to decrease the effective spin-mixing conductance of the $\mathrm{Pt} / \mathrm{CoFeB}$ interface. This directly improved the overall magnetic damping of the bilayer structure, which can be expressed as ${ }^{26}$

$$
\alpha=\alpha_{0}+\frac{\gamma \hbar^{2}}{2 e^{2} M_{\mathrm{s}} t_{\mathrm{FM}}} G_{\mathrm{eff}}^{\uparrow \downarrow},
$$

where $\alpha_{0}, G_{\text {eff }}^{\uparrow \downarrow}$, and $\gamma=1.88 \times 10^{11} \mathrm{~Hz} / \mathrm{T}$ are the intrinsic damping of the FM single layer, the effective spin-mixing conductance of the FM/HM interface, and the gyromagnetic ratio, respectively. $M_{\mathrm{s}}$ denotes the saturation magnetization, $t_{\mathrm{FM}}$ is the thickness of the FM layer, $\hbar$ is the reduced Planck constant, and $e$ is the electron charge. The precession of the magnetization in the FM layer results in a loss of spin angular momentum in the Pt layer, which acts as a spin sink. Thus, decreasing $G_{\text {eff }}^{\uparrow \downarrow}$ suppresses the pumping of spin from the FM to the HM, thus decreasing damping in the FM layer. In this letter, we study whether the effect of Hf dusting is also beneficial in the $\mathrm{NiFe} / \mathrm{Pt}$ system. We find that $\mathrm{Hf}$ dusting significantly improves damping and appears to slightly increase the $\mathrm{NiFe}$ effective magnetization, which is indicative of higher 
$\mathrm{NiFe}$ quality. However, for $\mathrm{Hf}$ thicker than $0.4 \mathrm{~nm}, \xi_{\mathrm{SH}}$ is found to decrease; as a consequence, we conclude that $0.4 \mathrm{~nm}$ is the optimum $\mathrm{Hf}$ thickness for reducing the expected threshold current for auto-oscillations. The additional benefit of a reduced damping will likely also enhance mutual synchronization in SHNO chains.

We prepared two stack series of $\mathrm{NiFe}(3) / \mathrm{Hf}\left(t_{\mathrm{Hf}}\right) / \mathrm{Pt}(5)$ and $\mathrm{NiFe}(5) / \mathrm{Hf}\left(t_{\mathrm{Hf}}\right) / \mathrm{Pt}(5)$ with $t_{\mathrm{Hf}}=0,0.4,0.5$, and 0.7 (all numbers are thicknesses in $\mathrm{nm}$ ) using the dc magnetron sputtering technique at room temperature onto high-resistive Silicon substrates. The base pressure was $3 \times 10^{-8}$ Torr, while the argon (Ar) plasma pressure was kept at 3 mTorr during deposition. $6 \mu \mathrm{m} \times 18 \mu \mathrm{m}$ bar-shape structures were then fabricated on the stacks using conventional optical lithography and $\mathrm{Ar}$ ion beam etching. Finally, coplanar waveguide structures were defined by $\mathrm{Cu} / \mathrm{Au}$ deposition and lift-off as the top electrodes. During processing, the sample with $t_{\mathrm{NiFe}}=3 \mathrm{~nm}$ and $t_{\mathrm{Hf}}=0.5 \mathrm{~nm}$ was lost; this did not impact any of the conclusions.

Figure 1(a) shows a schematic of the sample structure and our measurement setup. All measurements were performed at room temperature. In order to extract the anisotropic magnetoresistance (AMR) ratio of the stacks, we measured the magnetoresistance of each stack by sweeping the angle of the in-plane field from $\varphi=0^{\circ}$ to $360^{\circ}$ [shown in Fig. 1(a)], keeping its strength at $H_{\mathrm{IP}}=0.1 \mathrm{~T}$ while a dc current of $I_{\mathrm{dc}}=0.1 \mathrm{~mA}$ flowed through the microstripe. Figure 1(c) shows the magnetoresistance of the reference

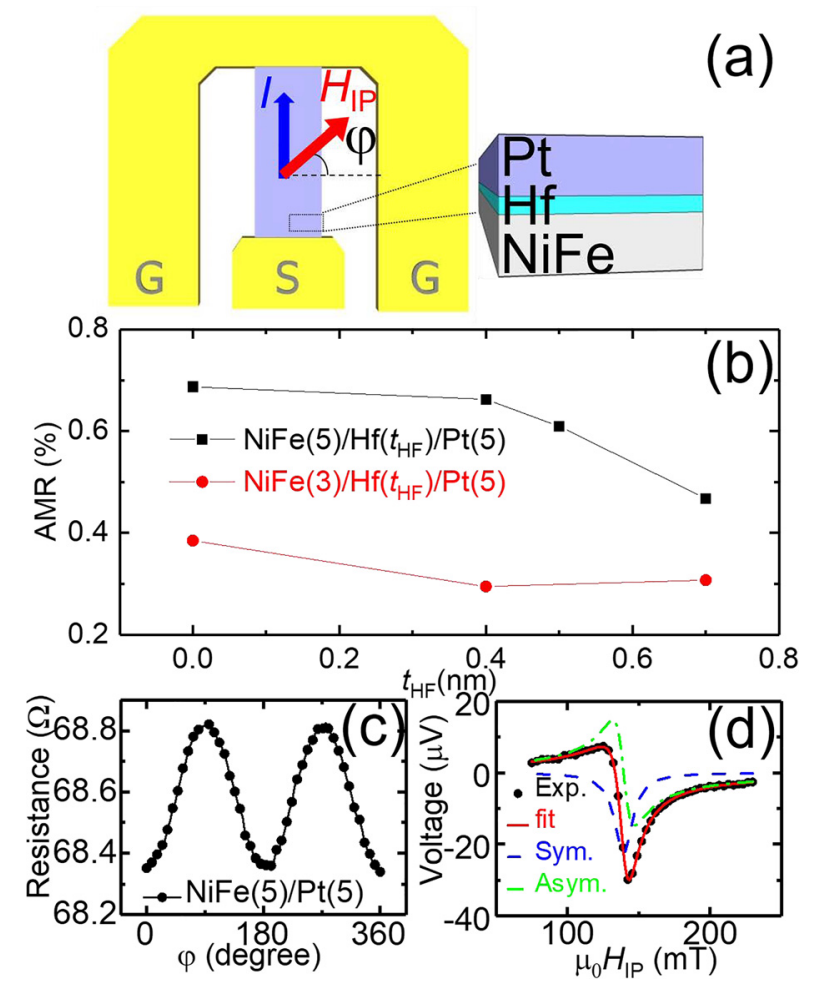

FIG. 1. (a) Schematic of the device, the material stack, and the direction of the direct current and of the in-plane field during the measurement. (b) Anisotropic magnetoresistance vs. Hf thickness for all stacks, measured in a rotating field of $H_{\mathrm{IP}}=0.1 \mathrm{~T}$ and a direct current $I_{\mathrm{dc}}=0.1 \mathrm{~mA}$. (c) Resistance vs. field angle for the reference sample $\mathrm{NiFe}(5) / \mathrm{Pt}(5)$. (d) ST-FMR Spectrum at $10 \mathrm{GHz}$ (black dots) for the same sample; the solid red line is a Lorentzian fit with the symmetric (blue) and asymmetric (green) terms shown separately. sample of $\mathrm{NiFe}(5) / \mathrm{Pt}(5)$ versus $\varphi$. The extracted AMR ratio for the stacks versus the thickness of the Hf layer $t_{\mathrm{Hf}}$ is shown in Fig. 1(b), where the AMR ratio drops as $t_{\mathrm{Hf}}$ increases.

In order to study the magnetodynamical properties of the stacks, we carried out spin-torque-induced ferromagnetic resonance (ST-FMR) measurements based on homodyne detection. ${ }^{27-31}$ For ST-FMR, we applied a pulse-modulated $15 \mathrm{dBm}$ microwave current alongside a dc drive current to the samples through a bias-tee. Between the rf source and the high-frequency port of the bias-tee, a microwave isolator was used to avoid any reflection of the microwave signal back towards the source. The output dc mixing voltage was then transmitted via the dc port of the bias-tee and subsequently measured using a lock-in amplifier. An in-plane magnetic field was applied at an angle of $\varphi=25^{\circ}$ and its strength $H_{\mathrm{IP}}$ was swept from $0.2 \mathrm{mT}$ to $0 \mathrm{mT}$, with the microwave frequency fixed during each sweep.

The ST-FMR spectra were measured for microwave frequencies from $2 \mathrm{GHz}$ to $12 \mathrm{GHz}$ for all samples with zero dc current, and each spectrum was fitted to a sum of a symmetric and an antisymmetric Lorentzian function ${ }^{32}$ [Fig. 1(d)]. Figure 2(a) shows different sets of the ST-FMR resonance frequency versus the in-plane field for all the samples (dots). All sets show good agreement with a fit to the Kittel formula (solid lines). ${ }^{33}$ The extracted effective magnetizations $\mu_{0} M_{\text {eff }}$ versus $t_{\mathrm{Hf}}$ are shown in Fig. 2(b), where we observe a slight improvement in the magnetization of the samples as a result of Hf dusting. The slight improvement might possibly be due to the reduction of the elastic strain from the Pt layer on $\mathrm{NiFe}^{25}$ The extracted ST-FMR linewidth values are shown in the inset to Fig. 2(a) (dots) with their fit to a linear function ${ }^{33}$

$$
\Delta H=\Delta H_{0}+4 \pi \alpha f / \gamma,
$$

where $\Delta H_{0}$ is the inhomogeneous broadening, $f$ is the STFMR resonance frequency, and $\alpha$ is the intrinsic damping of the magnetic layer. As we expected from Eq. (1), the extracted intrinsic damping shows a drop versus $t_{\mathrm{Hf}}$ [Fig. 2(c)], which is almost linear. Since the phenomenon is an interfacial effect, it is stronger for the sample set with thinner $\mathrm{NiFe}$, which can also be explained by Eq. (1), where the sample set with $3 \mathrm{~nm} \mathrm{NiFe}$ has a smaller value of the $M_{\mathrm{s}} t_{\mathrm{FM}}$ product. Figure 3 shows the damping versus $1 / \mu_{0} M_{\mathrm{s}} t_{\mathrm{FM}}$ for different $t_{\mathrm{Hf}}$ (dots). $M_{\mathrm{s}}$ is considered to be equal to the measured $M_{\text {eff }}$, since we expect negligible magnetocrystalline anisotropy for NiFe in our samples. By using these data in Eq. (1), we formed the following system of seven linear equations with five unknown parameters including $\alpha_{0}$ which is independent of $t_{\mathrm{Hf}}$ and $G_{\text {eff. } i}^{\uparrow \downarrow}$, with $i=1-4$ regarding $t_{\mathrm{Hf}}=0,0.4,0.5$, and $0.7 \mathrm{~nm}$, respectively:

$$
\left[\begin{array}{l}
0.0360 \\
0.0213 \\
0.0270 \\
0.0176 \\
0.0160 \\
0.0213 \\
0.0144
\end{array}\right]=\frac{\gamma \hbar^{2}}{2 e^{2}}\left[\begin{array}{ccccc}
1 & 0.61 & 0 & 0 & 0 \\
1 & 0.30 & 0 & 0 & 0 \\
1 & 0 & 0.57 & 0 & 0 \\
1 & 0 & 0.28 & 0 & 0 \\
1 & 0 & 0 & 0.29 & 0 \\
1 & 0 & 0 & 0 & 0.59 \\
1 & 0 & 0 & 0 & 0.29
\end{array}\right]\left[\begin{array}{c}
\alpha_{0} \\
G_{\text {eff }, 1}^{\uparrow \downarrow} \\
G_{\text {eff }, 2}^{\uparrow \downarrow} \\
G_{\text {eff }, 3}^{\uparrow \downarrow} \\
G_{\text {eff }, 4}^{\uparrow \downarrow}
\end{array}\right] .
$$




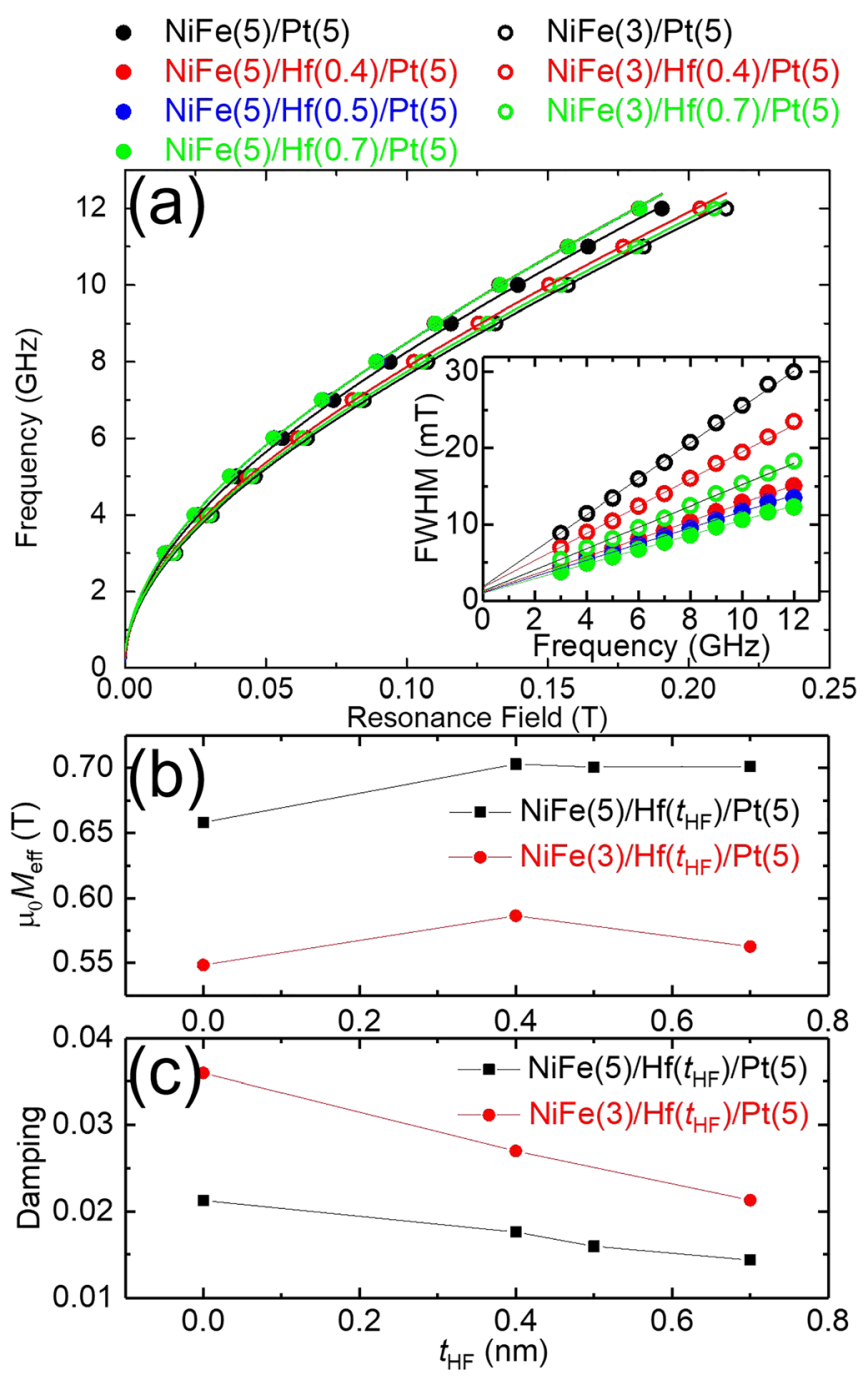

FIG. 2. (a) ST-FMR resonance frequency vs. field, with Kittel fits for all devices. Inset: ST-FMR linewidth vs. resonance frequency with linear fits. (b) $\mu_{0} M_{\text {eff }}$ vs. Hf thickness, extracted from the Kittel fits in (a). (c) Damping vs. the Hf thickness, as given by the slopes in the inset to (a).

Since our system of equations is overdetermined, we used the Least squares method to find the best solution of the unknown parameters. This results in $\alpha_{0}=0.0076 \pm 0.0005$, and the calculated effective spin-mixing conductance versus the Hf thickness is plotted in the inset of Fig. 3 where the error bars are the standard errors from the Least square method. As a consequence of the presence of the Hf layer at the interface, $G_{\mathrm{eff}}^{\uparrow \downarrow}$ of the FM/HM interface drops to almost $50 \%$ for $t_{\mathrm{Hf}}=0.7 \mathrm{~nm}$.

In order to measure the spin Hall efficiency for the stacks, a set of ST-FMR measurements were carried out on each sample: we swept the magnetic field at different current levels for negative and positive magnetic field polarities at a fixed microwave frequency of $5 \mathrm{GHz}$. The extracted STFMR linewidth versus dc current for the reference sample is shown in the inset to Fig. 4(a) (dots). We fit the linewidth for both field polarities to a linear function. The extracted slope of the lines, $\beta$, shows the rate of the linewidth changes over the dc current [Fig. 4(a)]. We observed a drop in $\beta$ as $t_{\mathrm{Hf}}$ increased. This was expected because, for thicker Hf, spin memory loss at the FM/HM interface attenuates the transmission of the generated spin to the magnetic layer. ${ }^{25,34}$

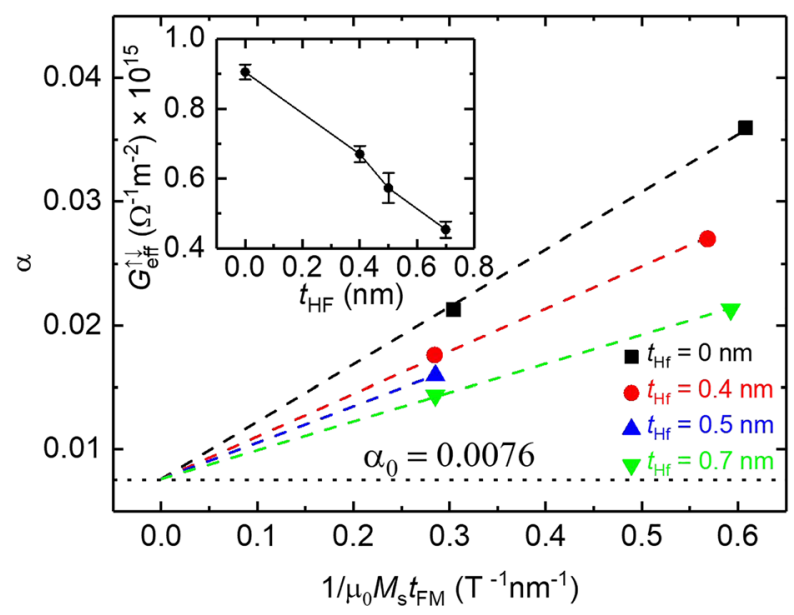

FIG. 3. Damping vs. $1 / \mu_{0} M_{\mathrm{s}} t_{\mathrm{FM}}$ (dots) for different Hf thicknesses; the inset shows the calculated $G_{\text {eff }}^{\uparrow \downarrow}$ vs. Hf thickness. The dashed lines are guidelines to Eq. (1) plotted using the calculated effective spin-mixing conductance.

Having extracted the parameters for each sample, we calculate the spin torque efficiency using the equation $32,35,36$

$$
\xi_{\mathrm{SH}}=\frac{\beta}{\frac{2 \pi f}{\gamma} \frac{\sin \varphi}{\left(H_{\mathrm{IP}}+0.5 M_{\mathrm{eff}}\right) \mu_{0} M_{\mathrm{s}} t_{\mathrm{FM}}} \frac{\hbar}{2 e}} \frac{R_{\mathrm{tot}}}{R_{\mathrm{NiFe}}} A_{\mathrm{C}},
$$

where $R_{\mathrm{NiFe}}$ and $R_{\mathrm{tot}}$ are the resistance of the NiFe layer and the whole stack, respectively, and $A_{\mathrm{C}}$ is the cross-sectional area of the measured microstrip. Figure 4(b) shows the calculated $\xi_{\mathrm{SH}}$ vs. Hf thickness, where thicker Hf results in lower spin torque efficiency. $\xi_{\mathrm{SH}}$ is almost independent of the thickness of the FM layer, which means that the torque on the FM layer from SHE is a damping-like torque and the field-like torque is negligible in our samples. ${ }^{23,34}$ Using $\xi_{\mathrm{SH}}$ for the reference sample, we estimate the internal spin Hall angle of Pt to be $\theta_{\mathrm{SH}}=0.22$ in the absence of significant spin memory loss, ${ }^{23,25}$ by using $\rho_{\mathrm{Pt}}=24 \mu \Omega \cdot \mathrm{cm}$ for the resistivity and $\lambda_{\mathrm{s}}^{\mathrm{Pt}}=1.2 \mathrm{~nm}$ for the spin attenuation length of $\mathrm{Pt}^{37,38}$ Our estimated value for $\theta_{\mathrm{SH}}$ is reasonable compared to previous reports. ${ }^{11,25}$

Finally, to get an approximate estimate of the effect of $\mathrm{Hf}$ on the threshold current of actual SHNOs, we extrapolate the plots of $\Delta H$ vs. $I_{\mathrm{dc}}$ [inset to Fig. 2(a)] to zero linewidth for each device; we get $J_{\text {th }}=2.56 \times 10^{7}$ and $3.88 \times 10^{7} \mathrm{~A} / \mathrm{cm}^{2}$ for $\mathrm{NiFe}(3) / \mathrm{Pt}(5)$ and $\mathrm{NiFe}(5) / \mathrm{Pt}(5)$, respectively. We then normalize $J_{\text {th }}$ of all other devices with these respective values and plot the result vs. $t_{\mathrm{Hf}}$ [Fig. $\left.4(\mathrm{c})\right]$. $J_{\text {th }}$ in both sample sets first goes down and then rises again for samples with thicker $\mathrm{Hf}$, with a minimum at $t_{\mathrm{Hf}}=0.4 \mathrm{~nm}$. This is due to the fact that the threshold current depends on both $\alpha$ and $\xi_{\mathrm{SH}}$, which act in opposite directions. While $\alpha$ shows a linear drop with $t_{\mathrm{Hf}}, \xi_{\mathrm{SH}}$ essentially remains constant to $t_{\mathrm{Hf}}=0.4 \mathrm{~nm}$ and then falls steeply, with the end result that $J_{\text {th }}$ reaches a minimum at $0.4 \mathrm{~nm}$

In conclusion, we investigated the effect of Hf dusting in $\mathrm{NiFe} / \mathrm{Pt}$ bilayer structures. We achieved a significant reduction in the intrinsic damping due to the decrease in the effective spin-mixing conductance of the NiFe/Pt interface. The NiFe effective magnetization also increased slightly in the presence of Hf. On the other hand, the spin Hall 


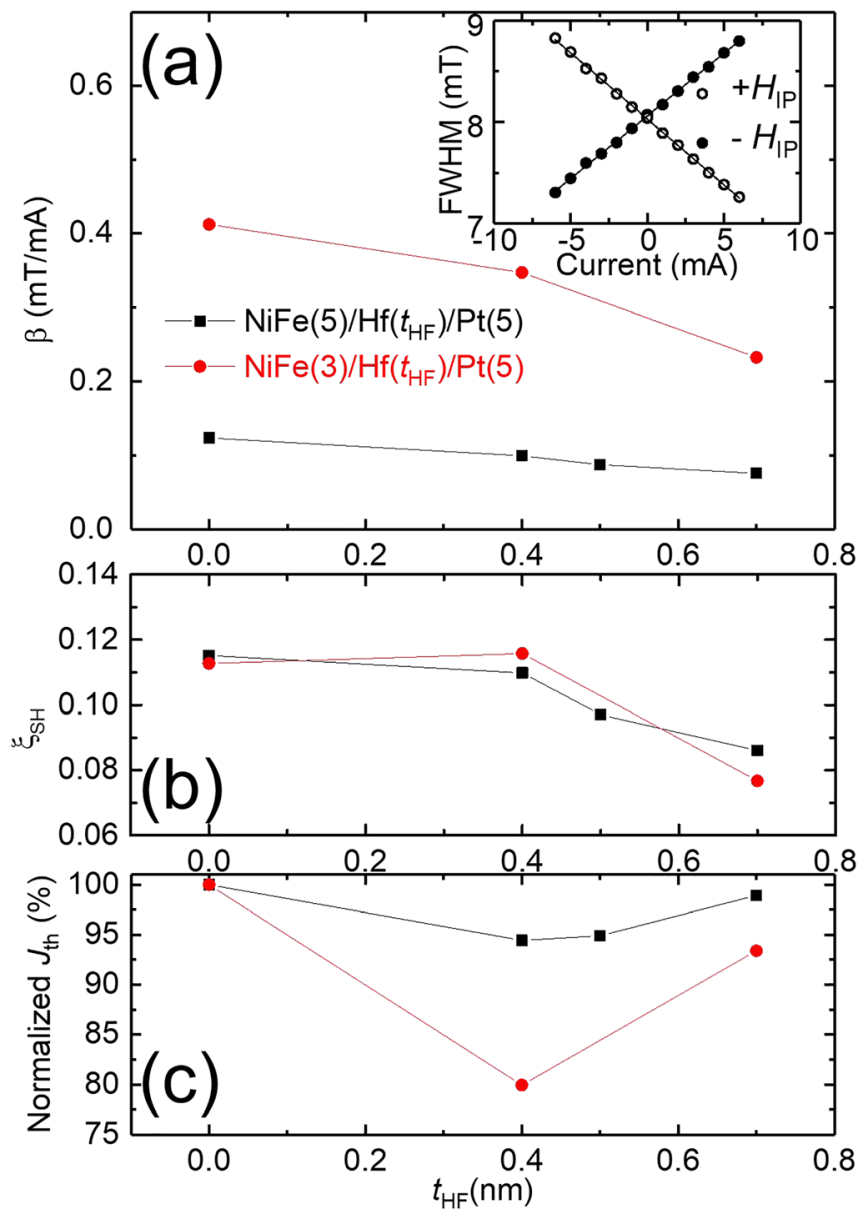

FIG. 4. (a) $\beta$ vs. Hf thickness. The inset shows linewidth vs. $I_{\mathrm{dc}}$ for $\mathrm{NiFe}(5) /$ $\mathrm{Pt}(5)$. (b) The calculated spin-Hall efficiency vs. Hf thickness and (c) the estimated auto-oscillation threshold current density normalized with the value for the samples without $\mathrm{Hf}$.

efficiency dropped rapidly for Hf thicker than $0.4 \mathrm{~nm}$. These opposite trends resulted in an optimum Hf thickness of $0.4 \mathrm{~nm}$ for the threshold current, for which an estimated reduction of up to $20 \%$ in $J_{\text {th }}$ could potentially be realized.

This work was supported by the Swedish Foundation for Strategic Research (SSF), the Swedish Research Council (VR), and the Knut and Alice Wallenberg Foundation (KAW). This work was also supported by the European Research Council (ERC) under the European Community's Seventh Framework Programme (No. FP/2007-2013)/ERC Grant No. 307144 "MUSTANG."

${ }^{1}$ J. E. Hirsch, Phys. Rev. Lett. 83, 1834 (1999).

${ }^{2}$ S. Zhang, Phys. Rev. Lett. 85, 393 (2000).

${ }^{3}$ Y. K. Kato, R. C. Myers, A. C. Gossard, and D. D. Awschalom, Science 306, 1910 (2004).

${ }^{4}$ E. Saitoh, M. Ueda, H. Miyajima, and G. Tatara, Appl. Phys. Lett. 88, 182509 (2006).

${ }^{5}$ S. O. Valenzuela and M. Tinkham, Nature 442, 176 (2006).

${ }^{6}$ V. E. Demidov, S. Urazhdin, A. Zholud, A. V. Sadovnikov, and S. O. Demokritov, Appl. Phys. Lett. 105, 172410 (2014).
${ }^{7}$ T. Chen, R. K. Dumas, A. Eklund, P. K. Muduli, A. Houshang, A. A. Awad, P. Dürrenfeld, B. G. Malm, A. Rusu, and J. Åkerman, Proc. IEEE 104, 1919-1945 (2016).

${ }^{8}$ P. Durrenfeld, A. A. Awad, A. Houshang, R. K. Dumas, and J. Åkerman, Nanoscale 9, 1285-1291 (2017).

${ }^{9}$ P. Dürrenfeld, F. Gerhard, M. Ranjbar, C. Gould, L. W. Molenkamp, and J. Åkerman, J. Appl. Phys 117, 17E103 (2015).

${ }^{10}$ M. Ranjbar, P. Dürrenfeld, M. Haidar, E. Iacocca, M. Balinskiy, T. Q. Le, M. Fazlali, A. Houshang, A. A. Awad, R. K. Dumas, and J. Åkerman, IEEE Magn. Lett. 5, 3000504 (2014).

${ }^{11}$ W. Zhang, W. Han, X. Jiang, S.-H. Yang, and S. S. P. Parkin, Nat. Phys. 11, 496-502 (2015).

${ }^{12}$ W. Skowroński, S. Zitek, M. Cecot, T. Stobiecki, J. Wrona, K. Yakushiji, T. Nozaki, H. Kubota, and S. Yuasa, in 2016 21st International Conference on Microwave, Radar and Wireless Communications (MIKON) (2016), pp. 1-3.

${ }^{13}$ M. Zahedinejad, A. A. Awad, P. Dürrenfeld, A. Houshang, Y. Yin, P. K. Muduli, and J. Åkerman, IEEE Magn. Lett. 8, 3704804 (2017).

${ }^{14}$ D. Fan, S. Maji, K. Yogendra, M. Sharad, and K. Roy, IEEE Trans, Nanotechnol. 14, 1083-1093 (2015).

${ }^{15}$ T. Kendziorczyk and T. Kuhn, Phys. Rev. B 93, 134413 (2016).

${ }^{16}$ A. A. Awad, P. Dürrenfeld, A. Houshang, M. Dvornik, E. Iacocca, R. K. Dumas, and J. Åkerman, Nat. Phys. 13, 292 (2017).

${ }^{17}$ M. Dvornik, A. A. Awad, and J. Åkerman, Phys. Rev. Appl. 9, 014017 (2018).

${ }^{18}$ H. Mazraati, S. Chung, A. Houshang, M. Dvornik, L. Piazza, F. Qejvanaj, S. Jiang, T. Q. Le, J. Weissenrieder, and J. Åkerman, Appl. Phys. Lett. 109, 242402 (2016).

${ }^{19}$ M. Zahedinejad, H. Mazraati, H. Fulara, J. Yue, S. Jiang, A. A. Awad, and J. Åkerman, Appl. Phys. Lett. 112, 132404 (2018).

${ }^{20}$ C.-F. Pai, L. Liu, Y. Li, H. W. Tseng, D. C. Ralph, and R. A. Buhrman, Appl. Phys. Lett. 101, 122404 (2012).

${ }^{21}$ L. Liu, C.-F. Pai, Y. Li, H. W. Tseng, D. C. Ralph, and R. A. Buhrman, Science 336, 555 (2012).

${ }^{22}$ T. Wang, W. Wang, Y. Xie, M. A. Warsi, J. Wu, Y. Chen, V. O. Lorenz, X. Fan, and J. Q. Xiao, Sci. Rep. 7, 1306 (2017).

${ }^{23}$ C.-F. Pai, Y. Ou, L. H. Vilela-Leão, D. C. Ralph, and R. A. Buhrman, Phys. Rev. B 92, 064426 (2015).

${ }^{24}$ S. Shi, Y. Ou, S. V. Aradhya, D. C. Ralph, and R. A. Buhrman, Phys. Rev. Appl. 9, 011002 (2018).

${ }^{25}$ M.-H. Nguyen, C.-F. Pai, K. X. Nguyen, D. A. Muller, D. C. Ralph, and R. A. Buhrman, Appl. Phys. Lett. 106, 222402 (2015).

${ }^{26}$ M. L. Polianski and P. W. Brouwer, Phys. Rev. Lett. 92, 026602 (2004).

${ }^{27}$ J. Sankey, P. Braganca, A. Garcia, I. Krivorotov, R. Buhrman, and D. Ralph, Phys. Rev. Lett. 96, 227601 (2006).

${ }^{28}$ J. C. Sankey, Y.-T. Cui, J. Z. Sun, J. C. Slonczewski, R. A. Buhrman, and D. C. Ralph, Nat. Phys. 4, 67 (2008).

${ }^{29}$ W. Chen, G. de Loubens, J.-M. L. Beaujour, J. Z. Sun, and A. D. Kent, Appl. Phys. Lett. 95, 172513 (2009).

${ }^{30}$ X. Cheng, J. A. Katine, G. E. Rowlands, and I. N. Krivorotov, Appl. Phys. Lett. 103, 082402 (2013).

${ }^{31}$ M. Collet, X. de Milly, O. d'Allivy Kelly, V. V. Naletov, R. Bernard, P. Bortolotti, J. Ben Youssef, V. E. Demidov, S. O. Demokritov, J. L. Prieto, M. Muñoz, V. Cros, A. Anane, G. de Loubens, and O. Klein, Nat. Commun. 7, 10377 (2016).

${ }^{32}$ L. Liu, T. Moriyama, D. Ralph, and R. Buhrman, Phys. Rev. Lett. 106, 036601 (2011).

${ }^{33}$ C. Kittel, Phys. Rev. 73, 155 (1948).

${ }^{34}$ Y. Ou, C.-F. Pai, S. Shi, D. C. Ralph, and R. A. Buhrman, Phys. Rev. B 94, 140414 (2016).

${ }^{35}$ K.-U. Demasius, T. Phung, W. Zhang, B. P. Hughes, S.-H. Yang, A. Kellock, W. Han, A. Pushp, and S. S. P. Parkin, Nat. Commun. 7, 10644 (2016).

${ }^{36}$ K. Ando, S. Takahashi, K. Harii, K. Sasage, J. Ieda, S. Maekawa, and E. Saitoh, Phys. Rev. Lett. 101, 036601 (2008).

${ }^{37}$ L. Liu, R. A. Buhrman, and D. C. Ralph, preprint arXiv:1111.3702 [condmat.mes-hall] (2011).

${ }^{38}$ W. Zhang, V. Vlaminck, J. E. Pearson, R. Divan, S. D. Bader, and A. Hoffmann, Appl. Phys. Lett. 103, 242414 (2013). 